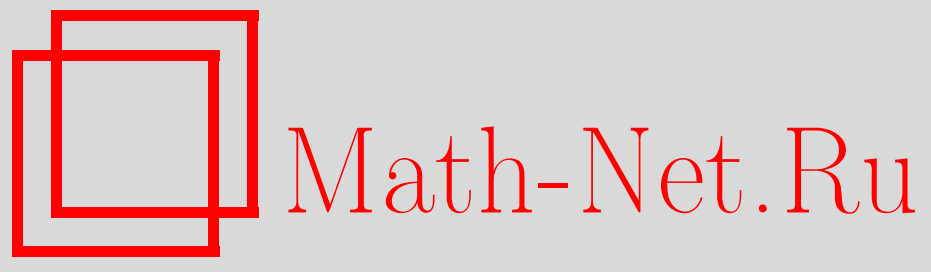

В. П. Маслов, О числе собственных значений для гиббсовского ансамбля самосопряженных операторов, $\mathrm{Ma-}$ тем. заметки, 2008, том 83, выпуск 3, 465-467

DOI: https://doi.org/10.4213/mzm4530

Использование Общероссийского математического портала Math-Net.Ru подразумевает, что вы прочитали и согласны с пользовательским соглашением http: //www . mathnet.ru/rus/agreement

Параметры загрузки:

IP: 54.84 .234 .179

26 апреля 2023 г., 15:35:45

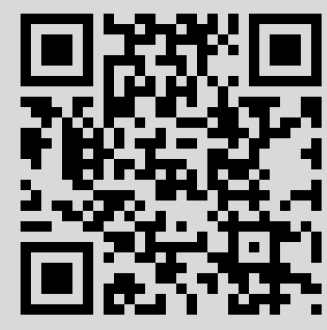




\section{О числе собственных значений для гиббсовского ансамбля самосопряженных операторов}

\section{В. П. Маслов}

Рассмотрим самосопряженный оператор $\widehat{H}$ с дискретным положительным спектром, с собственными значениями $\lambda_{1} \leqslant \lambda_{2} \leqslant \cdots \leqslant \lambda_{n} \leqslant \cdots, \lambda_{n} \rightarrow \infty$ при $n \rightarrow \infty$, и проекторами на соответствующие собственные подпространства $P_{1}, P_{2}, \ldots, P_{n}, \ldots$ в гильбертовом пространстве $L_{2}=L_{2}\left(\mathbb{R}^{D}\right)$, где $D$ - любое конечное целое. Будем предполагать, что для числа собственных значений, меньших заданного $E$ при $E \rightarrow \infty$, выполняется правило Вейля (в физике говорят, что число собственных значений пропорционально фазовому объему). Иногда эту формулу называют формулой Куранта.

Рассмотрим тензорное произведение $N$ гильбертовых пространств

$$
\mathscr{L}_{2}=L_{2}\left(\mathbb{R}^{D}\right) \otimes L_{2}\left(\mathbb{R}^{D}\right) \otimes L_{2}\left(\mathbb{R}^{D}\right) \otimes \cdots \otimes L_{2}\left(\mathbb{R}^{D}\right) \quad(N \text { раз }),
$$

где $N \rightarrow \infty$, и оператор

$\widehat{H}_{N}=[\widehat{H} \otimes 1 \otimes 1 \otimes \cdots \otimes 1]+[1 \otimes \widehat{H} \otimes 1 \otimes \cdots \otimes 1]+\cdots+[1 \otimes 1 \otimes \cdots \otimes 1 \otimes \widehat{H}] \quad(N$ слагаемых $)$ в нем.

Уравнение

с начальными условиями вида

$$
i \frac{\partial \Psi}{\partial t}=\widehat{H} \Psi
$$

$$
\left.\Psi\right|_{t}=\varphi_{s}^{(i)}(x), \quad \varphi_{s}^{(i)}(x) \in L_{2}\left(\mathbb{R}^{D}\right), \quad x \in \mathbb{R}^{D},
$$

где $i=1,2, \ldots, N$, такими, что последовательность $\varphi_{s}^{(i)}(x)$ при $s \rightarrow \infty$ слабо сходится к нулю, $\left\|\varphi_{s}^{(i)}(x)\right\|_{L_{2}\left(R^{k}\right)}=1$, а $E_{s}^{(i)}=\left(\varphi_{s}^{(i)}(x), \widehat{H} \varphi_{s}^{(i)}(x)\right) \rightarrow \infty$ при $n \rightarrow \infty, E_{s}^{(i)} \leqslant E, P_{n} \varphi_{s}^{(i)}=0$, если $\lambda_{n}>E$.

Соответственно,

с начальным условием вида

$$
i \frac{\partial \Psi_{N}}{\partial t}=\widehat{H}_{N} \Psi_{N}, \quad \Psi_{N} \in \mathscr{L}_{2}
$$

$$
\left.\Psi_{N}\right|_{t=0}=\varphi_{s}^{(1)}\left(x_{1}\right) \varphi_{s}^{(2)}\left(x_{2}\right) \cdots \varphi_{s}^{(N)}\left(x_{N}\right), \quad x_{i} \in \mathbb{R}^{D},
$$

при $N \rightarrow \infty, n \rightarrow \infty$ будем называть гиббсовским ансамблем.

Если $\varphi_{s}^{(k)}\left(x_{k}\right)$ не равны друг другу при $k=1, \ldots, N$, то мы определим параметр $b_{E}$ из условия

$$
\frac{\operatorname{Sp}\left(\widehat{H} e^{-b_{E} \widehat{H}}\right)}{\operatorname{Sp}\left(e^{-b_{E} \widehat{H}}\right)}=E .
$$

Обозначим $N_{0}=\mathrm{Sp} e^{-b_{E} \widehat{H}}$. Числа заполнения оператора $\widehat{H}_{N}$, отвечающие собственной функции $\Psi_{i}$ оператора $\hat{H}$, меньшие или равные $N E$, будем обозначать $N_{i}$. Сумму $N_{i}+N_{i+1}+\cdots+N_{i+k}$ обозначим через $N_{i}^{(k)}$ - числа заполнения, отвечающие набору $\Psi_{i}, \Psi_{i+1}, \ldots, \Psi_{i+n}$, а проектор на подпространство, натянутое на собственные подпространства, отвечающие им, через $P_{i}^{(k)}$.

Для несимметричного гиббсовского ансамбля имеет место теорема.

Теорема 1. Существуют такие постоянные $C_{l}, l=1,2, \ldots$, что для любых $i>1 u$ $k>0$ числа заполнения $N_{i}^{(k)}$, удовлетв оряющие неравенству

$$
\mathrm{P}\left(\left|N_{i}^{(k)}-B \operatorname{Sp}\left(P_{i}^{(k)} e^{-b_{E} \widehat{H}}\right)\right|>B \sqrt{N_{0} \ln N_{0}} \psi\left(N_{0}\right)\right) \leqslant C_{l} N_{0}^{-l}, \quad l=1,2, \ldots, \quad B=N / N_{0},
$$

(C) В. П. МАслов, 2008 
где $\psi(x)$ - положительная функиия, произвольно медленно стремящаяся $\kappa+\infty$ при $x \rightarrow+\infty, a \mathrm{P}(\cdot)$ - отношение числа собственных значений гамильтониана $\widehat{H}_{N}$, не превосходящих NE и удовлетворяющих стоящему в скобках неравенству, к общему числу собственных значений гамилътониана $\widehat{H}_{N}$, не превосходящих $N E$.

Если начальное условие (3) симметрично (т.е. $\varphi_{s}^{(k)}(x)=\varphi_{s}^{(m)}(x)$ при всех $k$ и $m$ ), то оно разлагается только по симметрическим собственным функциям оператора $\widehat{H}_{N}$. Выражение вида $\sum_{i=1}^{k} \lambda_{i} N_{i}$ для любых $N_{i}$ таких, что $\sum_{i=1}^{k} N_{i} \leqslant N$, есть собственное значение $\widehat{H}_{N}$, отвечающее симметричным по $\left(x_{i}, x_{j}\right)$ собственным функциям. Снова обозначим $N_{i}^{(k)}=\sum_{j=i}^{i+k} N_{j}$. Будем называть эту задачу симметричным гиббсовским ансамблем.

Пусть $b_{E, N}-$ решение уравнения

$$
\operatorname{Sp}\left(\frac{\widehat{H}}{e^{b_{E, N} \widehat{H}}-1}\right)=E \operatorname{Sp}\left(\frac{1}{e^{b_{E, N} \widehat{H}}-1}\right)+\lambda_{1}\left(N-\operatorname{Sp}\left(\frac{1}{e^{b_{E, N} \widehat{H}}-1}\right)\right) .
$$

Тогда для "почти всех" чисел заполнения $N_{i}^{(k)}$ при $k \rightarrow \infty$ имеет место равенство

$$
N_{i}^{(k)} \cong \operatorname{Sp}\left(P_{i}^{(k)} \frac{1}{e^{b_{E, N} \widehat{H}}-1}\right),
$$

где $P_{i}^{(k)}=P_{i}+P_{i+1}+\cdots+P_{i+k}-$ проектор на подпространство, порожденное подпространствами $P_{i} L_{2}, P_{i+1} L_{2}, \ldots, P_{i+k} L_{2}$.

Более точно утверждение формулируется следующим образом.

Теорема 2. Существуют такие постоянные $C_{l}, l=1,2, \ldots$, что для любых $i>1 u$ $k>0$ числа заполнения $N_{i}^{k}$, удовлетворяющие неравенству

$$
\mathrm{P}\left(\left|N_{i}^{(k)}-\operatorname{Sp}\left(P_{i}^{(k)} \frac{1}{e^{b_{E}, N} \hat{H}-1}\right)\right|>\sqrt{\widetilde{N}_{0} \ln \widetilde{N}_{0}} \psi\left(N_{0}\right)\right) \leqslant C_{l} \widetilde{N}_{0}^{-l}, \quad l=1,2, \ldots,
$$

где

$$
\tilde{N}_{0}=\operatorname{Sp}\left(\frac{1}{e^{b_{E, N} \hat{H}}-1}\right)
$$

$u$ где $\psi(x)$ - положительная функиия, произвольно медленно стремящаяся $\kappa+\infty$ при $x \rightarrow+\infty, a \mathrm{P}(\cdot)$ - отношение числа собственных значений гамильтониана $\widehat{H}_{N}$, отвечающих симметричным по $\left(x_{i}, x_{j}\right)$ собственным функииям, не превосходящих $N E$ u удовлетворяющих стоящему в скобках неравенству, к общему числу собственных значений гамильтониана $\widehat{H}_{N}$, не превосходящих $N E$ и отвечающих симметричным собственным функииям.

Таким образом, в симметрическом случае $\widetilde{N}_{0}$ зависит от $N$ и если $\widetilde{N}_{0}(N)<N$, то возникает "диффузия", при которой увеличивается кратность $\lambda_{1}$ на величину $N-\widetilde{N}_{0}(N)$, т.е. при $N>a \widetilde{N}_{0}(N), a>1$ возникает скопление собственных значений оператора $\widehat{H}_{N}$ на нижнем уровне $\lambda_{1}$ (конденсат).

Пусть $\widehat{H}=H(\widehat{p}, q)$ оператор в $L_{2}\left(\mathbb{R}^{n}\right), \widehat{p}=i h \partial / \partial q$, где $H(p, q)$ - символ Вейля (или Иордана).

Пусть символ гамильтониана $H(p, q)$ есть выпуклая функция, растущая не медленнее, чем $|p|+|q|$, и не быстрее, чем $|p|^{s}+|q|^{s}$, где $s$ - любое целое. Тогда можно перейти к системе координат $H(p, q)=$ const и нормальным к ним $\vec{n}$. Сделав замену $\vec{l}=b \vec{n}$, мы получим, что

$$
\frac{\iint H(p, q) e^{-b H(p, q)} d p d q}{\iint e^{-b H(p, q)} d p d q}=\frac{1}{b} \frac{\iint H(p, q) e^{-H(p, q)} d p d q}{\iint e^{-H(p, q)} d p d q}=E, \quad N_{0} \cong \iint e^{-b H(p, q)} d p d q .
$$

Причем нетрудно убедиться, что $a_{1} b^{-n} \geqslant N_{0} \geqslant a_{2} b^{-2 n / s}$, где $a_{1}$ и $a_{2}$ - некоторые константы. 
Кроме того, при этих ограничениях

$$
\operatorname{Sp}\left(e^{-H(\widehat{p}, q)}\right) \stackrel{h \rightarrow 0}{\longrightarrow} \iint e^{-H(p, q)} d p d q
$$

а число собственных значений, меньших $\lambda$ при $\lambda \rightarrow \infty$, приближенно равно фазовому объему $H(p, q) \leqslant E$, деленному на $h^{n}$ (см. [1], [2]).

Следовательно, при $b \rightarrow 0$ условия $E \rightarrow \infty, N_{0} \rightarrow \infty$ выполнены, и мы приходим после перехода к пределу при $h / b \rightarrow 0$ к следующему.

Плотность спектра при $h \rightarrow 0$ переходит в отношение данного фазового объема к фазовому объему, определяемому неравенством

$$
\sum_{i=1}^{N_{0}} H\left(p_{i}, q_{i}\right) \leqslant N_{0} E
$$

Пусть $\rho_{E_{l}}^{E_{l+1}}-$ плотность “чисел заполнения" в интервале $E_{l}$ и $E_{l+1}$.

Разобьем фазовое пространство $p, q$ на конечное число областей $E_{l} \leqslant H(p, q) \leqslant E_{l+1}$, $l=0, \ldots, s-1, E_{0}=0, E_{s}=E ; p, q \in \mathbb{R}^{n}$ и соответственно фазовое пространство $p_{1}, q_{1}, p_{2}, q_{2}, \ldots, p_{N}, q_{N}$. Производя выборку с возвращением $N_{l}$ из $2 n N$ областей в "ящик" $E_{l} \leqslant H(p, q) \leqslant E_{l+1}$ (в гиббсовском случае упорядоченную, а в бозевском случае неупорядоченную), при условии (10) мы получим $\rho_{E_{l}}^{E_{l+1}}=N_{l} / N$ [3] и теорему в независимой от допредельного квантового варианта формулировке. В нижеприводимой теореме $\mathrm{P}$ есть лебегова мера фазового объема, определенного в скобках (11), относительно всего объема (10).

Теорема 3. В гиббсовском случае имеет место неравенство

$$
\mathrm{P}\left(N_{0} \rho_{E_{l}}^{E_{l+1}}-\iint_{E_{l} \leqslant H(p, q) \leqslant E_{l+1}} e^{-b H(p, q)} d p d q \geqslant \sqrt{N_{0} \ln N_{0}} \Psi\left(N_{0}\right)\right) \leqslant N_{0}^{-m},
$$

где $m$ - любое целое.

Здесь мы не используем, как в случае классической теоремы Козлова [4; с. 143], гипотезу Гиббса, поэтому аналогичная теорема имеет место в неупорядоченном (бозевском) случае.

Приношу глубокую благодарность В. Е. Назайкинскому за плодотворные обсуждения и В. Е. Подольскому за полезные консультации.

\section{СПИСОК ЦИТИРОВАННОЙ ЛИТЕРАТУРЫ}

[1] B. Helffer, Théorie Spectrale pour des Operateurs Globalement Elliptiques, Astérisque, 112, Soc. Math. France, Paris, 1984. [2] Ф.А. Березин, М. А. Шубин, Уравнение Шредингера, Изд-во МГУ, М., 1983. [3] А.Н. Ширяев, Вероятность, кн. 1: Элементарная теория вероятностей. Математические основания. Предельные теоремы, МЦНМО, М., 2004. [4] В. В. Козлов, Тепловое равновесие по Гиббсу и Пуанкаре, Современная математика, Ин-т компьютерных исследований, М.-Ижевск, 2002.

\section{В. П. Маслов}

Поступило

Московский государственный 07.10.2007 университет им. М. В. Ломоносова

E-mail: v.p.maslov@mail.ru 